\title{
Introduction
}

Karina Aveyard Griffith University

\author{
Author Ms Karina Aveyard \\ School of Humanities \\ Griffith University, Nathan Campus \\ 170 Kessels Road \\ Nathan QLD 4111, Australia \\ Karina.Aveyard@student.griffith.edu.au
}

\section{Contributor details}

Karina Aveyard is a Ph.D. candidate at the School of Humanities at Griffith University. Her doctoral research examines contemporary cinema exhibition in regional and rural locations. This research is funded by an Australian Research Council Linkage Grant in partnership with the Australian government film agencies the National Film and Sound Archive and Screen Australia.

This themed issue of Studies in Australasian Cinema addresses how digital technologies are re-shaping the cinematic landscape in Australia and New Zealand, and beyond. Collectively the articles explore the impact of digitization from a range of perspectives encompassing the transnational to the local, and various industrial spheres including independent and experimental film production, distribution and exhibition. The digital theme is continued into the new 'VaultAge' section of the journal, which, in this issue, profiles the online film archival resource, Australianscreen online, which is produced by the National Film and Sound Archive.

In practical terms, 'going digital' may be understood as a technological transition that has enabled the shift from the use of linear (analogue) formats, such as $35 \mathrm{~mm}$ film, video tape and optical special effects, to the use of formats based on non-linear (digital) data, such as DVD, Blu-ray, cinematic data projection and computergenerated special effects and imagery. However, being digital not only encompasses elements of technological evolution, but also of innovation - the Internet is an entirely new media and communications platform to which digital technologies have also given rise. While the Internet enables a wide variety of applications and uses, most significant for film-makers, content circulators and film audiences has been the establishment of a new medium for the distribution of cinematic material and a new 'screen' for watching film content. 
In his work on media convergence, Henry Jenkins draws a distinction between media platforms and the technologies that facilitate their delivery. The former encompassing the formats and protocols that enable communication, and the latter constituting the means by which content is delivered (Jenkins 2006: 13-14). In one sense it is possible to deconstruct the digital innovations in cinema along similar lines - cinema venues, televisions and the Internet constituting the platforms, and the other shifting analogueto-digital processes (as outlined above) representing modifications of existing creative and industrial practices, which facilitate rather than re-invent these cinema platforms. For example, audiences continue to watch films in cinemas but now these can be screened using data files and digital projectors rather than traditional 35mm. Similarly, the manipulation of film images to enhance dramatic effect is not something that has arisen entirely in the digital age but has been practised by filmmakers for almost as long as films have been made. The difference now is that rather than being facilitated by mechanical and photographically based optical techniques, the look of films is transformed on computers that use digital technologies to produce images and visual effects.

However, while these delineations may be useful in scoping some of the practical dimensions of this technological transition, they represent a rather limited framework through which to understand the impact and implications of digitization on cinema. Rather than being fulfilled through discrete, purpose-orientated functions, the implementation and use of digital technologies across creative practices, delivery technologies and end-user platforms are highly interconnected and interdependent. As the article by Ramon Lobato demonstrates, the success of commercial films distributed online continues, for the most part, to be determined by traditional distributor/corporate affiliations and is closely linked to the film's performance in the theatrical release window. Sean Cubitt explores the potential of the Internet as a platform with the capacity to facilitate a new interactive and more democratic dialogue between content creators and audiences that enriches and extends the cinematic experience. But as Cubitt demonstrates, the Internet is also a highly corporatized platform, controlled by governmental and industrial organizations and complex systems of standards, which serve to restrain and restrict as much as they might liberate. Shifting from the sphere of consumption to content creation, Adrian 
Martin's article profiling the work of experimental film-maker, James Clayden, explores the interrelationships, rather than oppositions, between digital and film-based modes and styles of production.

The articles in this issue also contribute to an evolving critical discourse that is framed beyond the technical, economic and legal arguments that, until recently, have tended to dominate scholarship dealing with the digital transition. A key focus of the articles in this issue is to interrogate the hitherto dominant globalized perspectives on digitization by exploring the forces that come into play within it, at the national and local levels, and by imagining what might exist outside it, in the realm of the transnational. By considering how digital technologies are being implemented and used in specific material situations, scholarship is opened to the constructive possibilities that arise from recognizing the interactive and, at times, contradictory relationships that exist between the global and other locations. Cinema histories have already been enriched by approaches to research that are similarly engaged with how social practices and institutional activities from within and outside globalized contexts have influenced the shape and meaning of cinema (see Studies in Australasian Cinema 1:3).

The issue begins with Sean Cubitt's examination of the sphere of transnational cinema, where digital technologies play an important role in facilitating and accelerating the development of cinema that extends beyond nations. Cubitt explores the potential for the development of an authentic alter-globalizing transnational sphere for cinema, which can be constituted only outside the restrictive infrastructures and standards of the current, actually existing transnational public sphere that is dominated by the agendas of governments, major corporations and civil society organizations. Examining three forms of cinema in Australia and Aotearoa New Zealand, Cubitt tests the possibilities for the development of a truly alter-global cinematic space. Against this he examines the role of digital technologies in the ongoing shift from biopolitical and commodity-based economies to the emerging database economy and the complex and contested processes of the development of technical standards. 
Ramon Lobato's article continues the focus on the online space in a critical examination of the myths surrounding the democratizing power of the Internet through the lens of the online video-on-demand industry. Lobato analyzes the various services that offer commercial (and legal) movie downloads and streaming on the Internet. In doing so, he demonstrates that rather than representing a break with the past, online delivery of film is beholden to market forces very similar to those that control more traditional distribution formats. This has implications for the range of films available to viewers and the conditions under which they are accessible, and challenges assumptions about the liberatory capabilities of the Internet.

Adrian Martin's article shifts the focus to production with his examination of the work of James Clayden, a Melbourne-based film-maker, painter, dramatist and performer. Active since the 1970s, Clayden's cinematic works span a range of formats from Super 8, 16mm, 35mm through to digital, and include several experimental features and numerous short films. Clayden's recent projects push the boundaries of the digital aesthetic as it is conventionally theorized through their innovative and, at times, radical combinations of audio-visual forms, narratives and styling. His work has particular relevance in the cross-over from film to digital and spaces in between, and it challenges notions about the disconnect between digital and film-based modes and aesthetics of production. Martin uses a single performance of two of Clayden's works, The Ghost Paintings 2 (2002, video) and the theatrical piece Macbeth $X$ (2002), as a framework through which to explore both the creativity and diversity of Clayden's work and to suggest a new approach to the theorization of the emerging digital aesthetics of Australian cinema.

Finally, my article examines the rapidly developing landscape of digital exhibition in Australia's commercial cinema industry. While the article outlines the major developments to date, its principal focus lies beyond the American studios and multiplexes. It examines how the transition is occurring outside the mainstream where the financial imperatives of digital cinema threaten the ongoing viability of many independently owned venues. Significant closures of independent cinemas would have a serious impact on the diversity of Australian cinematic culture by reducing the options for audience seeking an alternative to the multiplex experience and limiting their opportunities to see specialized and independent films. Of particular concern is 
the fate of independents in regional and rural locations, many of which provide the only public screening opportunities for film within large geographic areas.

Many thanks to each of the contributors to this issue - Sean Cubitt, Ramon Lobato and Adrian Martin - for their outstanding articles and for their helpful advice and encouragement. Many thanks also to Albert Moran, Wendy Keys, Deb Verhoeven and Ian Henderson, whose advice and assistance in the preparation of this issue is greatly appreciated. 